\title{
基于非对称性三唑萗啶吡啶铜催化合成吡嗪与酮衍生物
}

\author{
李家豪 $a$ 杨义科 $b$ 胡文康 $a$ 夏晓峰 $a$ 王大伟 $* a$ \\ ( ${ }^{a}$ 江南大学化学与材料工程学院 教育部合成与生物胶体重点实验室 江苏无锡 214122) \\ ( $b$ 河南警察学院刑事科学技术系 郑州 450046)
}

\begin{abstract}
摘要 发展了一种基于非对称性配体的催化剂 TNP-Cu@rGO, 并将该催化剂用于催化合成吡嗪和酮衍生物的新方法. 对该反应中碱的种类、溶剂、反应温度和时间等条件进行了优化，确定了最优的反应条件. 接着对该反应的底物普适 性进行了研究, 实验表明该催化体系显示了良好的官能团耐受性, 并对该催化体系和反应进行了机理研究和探索. 催 化剂的回收循环再利用实验表明, 该催化剂循环使用五次仍能保持较高的催化活性.
\end{abstract}

关键词 借氢; 非对称性; 吡嗪; 铜

\section{Catalytic Synthesis of Pyrazine and Ketone Derivatives by Unsymmetrical Triazolyl-Naphthyridinyl-Pyridine Copper}

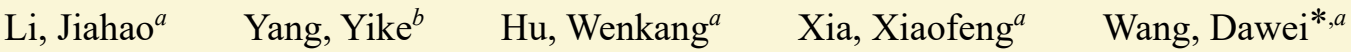 \\ ( ${ }^{a}$ Key Laboratory of Synthetic and Biological Colloids, Ministry of Education, School of Chemical and \\ Material Engineering, Jiangnan University, Wuxi, Jiangsu 214122) \\ ( ${ }^{b}$ Department of Criminal Science and Technology, Henan Police College, Zhengzhou 450046)
}

\begin{abstract}
A novel method for the synthesis of pyrazine and ketone derivatives was developed by using asymmetric ligand-based catalyst TNP-Cu@rGO. The screening of reaction bases, solvents, reaction temperatures and time was conducted to determine optimal reaction conditions. The reaction scope and generality of this methodology for the synthesis of pyrazines were tested and the results showed that this catalytic system had good functional tolerance. More than twenty substrates experiments were successfully set up, and pyrazines with moderate to high yields were isolated. Additionally, this catalytic system could also be used to the systhesis of ketones in good yields. Furthermore, the mechanism investigations were conducted for the synthesis of pyrazine derivatives to better understand the synthesis of pyrazines. The recycling test of TNP-Cu@rGO for the synthesis of pyrazines was investigated and the experiments showed that the catalyst could still maintain high catalytic activity for five times.
\end{abstract}

Keywords borrowing hydrogen; unsymmetrical; pyrazine; copper

杂环胺类化合物广泛存在于自然界中, 是一类重要 的有机化合物. 由于其数量庞大, 种类繁多, 且具有较 高的生物活性, 很多年来一直备受国内外科学家的重 视 ${ }^{[1]}$. 其中, 吡嗪类衍生物更是在农药、医药、功能材料、 食品添加剂等领域表现出优异的性能, 因而取得了较广 泛的应用 ${ }^{[2]}$. 另外, 吡嗪类药物由于具有抗菌、治疗心脑 血管疾病、降血脂降血糖等作用, 也在临床医学中发挥 重要的作用(Scheme 1) ${ }^{[3]}$.

随着科学家从食物中成功分离出烷基吡嗪化合物, 学者们开始探索吡嗪化合物的人工合成方法, 陆续成功

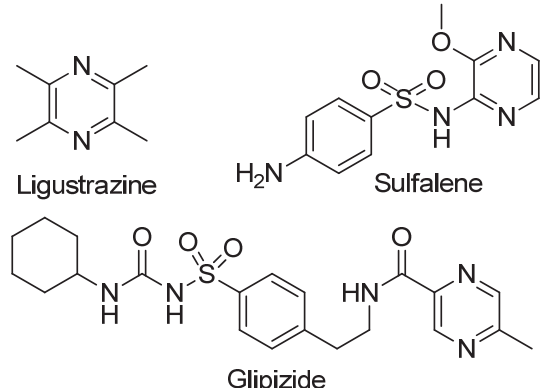

图式 1 吡嗪类活性药物

Scheme 1 Pyrazine active drugs

* Corresponding author. E-mail: wangdw@jiangnan.edu.cn

Received July 7, 2021; revised September 2, 2021; published online September 9, 2021.

Project supported by the National Natural Science Foundation of China (No. 21776111).

国家自然科学基金(No. 21776111)资助项目. 
合成了甲基吡嗪、2,6-二甲基吡嗪等吡嗪化合物. 近些 年由于烷基吡嗪化合物在医药、农药、光电材料等领域 的广泛应用, 再次引起了国内外研究者们的兴趣和关 注, 传统合成烷基吡嗪化合物主要采用卤代烃、醛酮等 烷基化试剂, 这些方法虽然能够得到烷基吡嗪产物, 但 往往需要较为苛刻的反应条件, 且会产生大量副产物, 从而造成环境污染, 因而限制了其应用 [4]. 醇类化合物 因其具有廉价、易制备、低毒性、烷基化后的副产物是 水等特点, 成为一种极具潜力的烷基化试剂, 因此受到 了科研工作者的青睐 ${ }^{[5]}$. 2012 年, Li 课题组 ${ }^{[6 a]}$ 报道了以 甲醇为烷基化试剂, 在 $\left[\mathrm{Cp}^{*} \mathrm{IrCl}_{2}\right]_{2} / \mathrm{NaOH}$ 体系下对芳香 伯胺进行 $N$-烷基化反应, 该反应具有催化剂用量低、适 用范围广等优点. 2017 年该课题组 ${ }^{[6 b]}$ 又制备了含 $N^{\wedge} \mathrm{N}$ 双齿配体的铱络合物 $\left(\left[\mathrm{Cp} * \operatorname{Ir}\left(\mathrm{BiBzImH} \mathrm{I}_{2}\right) \mathrm{Cl}\right][\mathrm{Cl}]\right)$, 并将 其再次用于该反应中, 该催化剂实现了在弱碱存在的条 件下杂环芳胺的顺利烷基化. 2018 年, Fujita 课题组 ${ }^{[6 c]}$ 合 成了一种氮杂卡宾铱催化剂, 将其运用至芳胺的 $N$-烷 基化反应中, 也获得了较高的反应收率. 2019 年, Namitharan 课题组 ${ }^{[6 \mathrm{~d}]}$ 运用商用 $\mathrm{Fe}_{2} \mathrm{O}_{3}$ 纳米粒子实现芳胺与 甲基酮的直接 $N$-烷基化与 $C$-烷基化合成芳胺和酮类衍 生物. 2020 年, Mannathan 课题组 ${ }^{[6 \mathrm{e}]}$ 报道了使用 $\mathrm{Zn}\left(\mathrm{NO}_{3}\right)_{2} \cdot 6 \mathrm{H}_{2} \mathrm{O}$ 为催化剂, 成功实现杂环芳胺的 $N$-烷基 化反应，并得到单烷基化的产物，收率较高.

其中, 在此转化过程中常用的 $\mathrm{Ru} 、 \mathrm{Ir} 、 \mathrm{Rh} 、 \mathrm{Pd}$ 等贵 金属作为催化剂的研究相对成熟, 能够得到较高的转化 率和产率 ${ }^{[7]}$. 近年来科研工作者开始积极探索使用廉价 金属 $\mathrm{Fe} 、 \mathrm{Mn} 、 \mathrm{Ni} 、 \mathrm{Cu}$ 等, 催化该类反应. 相对于贵金 属而言, 发展和利用价格低廉、来源广泛的廉价金属催 化剂, 更符合当今有机化学和精细化工的发展理念 ${ }^{[8]}$.

最近, 我们课题组 ${ }^{[9]}$ 报道了几种含氮、磷配体的金 属催化剂, 并将其成功应用到借氢反应和脱氢偶联反应 中. 然而, 这些催化剂大部分不能回收使用或者回收后 催化性能降低 ${ }^{[10]} .2019$ 年, 我们 ${ }^{[11]}$ 报道了一种基于非对 称性三唑萗啶吡啶配体铜 TNP-Cu@rGO 催化剂(TNP: Triazolyl-Naphthyridinyl-Pyridine), 能够实现脱氢偶联 反应合成喹啉衍生物, 并表现出较高的活性. 本文将 $\mathrm{TNP}-\mathrm{Cu} @ \mathrm{rGO}$ 成功应用到合成烷基吡嗪化合物与酮类 衍生物的反应中. 实验结果表明, 该催化剂对合成烷基 吡嗪化合物和酮类衍生物表现出优异的催化活性, 相较 于之前文献所报道方法, 该催化剂具有高活性、可回收 等优点. TNP-Cu@rGO 对于合成烷基吡嗪类化合物具有 更好的底物普适性. 最后通过控制实验及对中间体的捕 获, 研究了该催化体系的反应历程, 并提出了可能的反 应机理, 同时也实现了该催化剂的回收再利用实验.

\section{1 结果与讨论}

非均相催化剂 TNP-Cu@rGO 和配体(TNP)是按照 已知方法合成, 具体见参考文献合成 ${ }^{[11]}$.

\section{1 反应条件的优化}

选择氨基吡嗪和苄醇作为模型底物, TNP-Cu@Go 作为催化剂尝试烷基吡嗪衍生物的合成反应(如表 1 所 示). 首先, 考察碱对反应的影响, 实验结果表明当使用 碱性较弱的碱时, 例如: $\mathrm{K}_{2} \mathrm{CO}_{3} 、 \mathrm{Na}_{2} \mathrm{CO}_{3} 、 \mathrm{~K}_{3} \mathrm{PO}_{4}$, 反应 无法进行或者获得较低的反应收率(Table 1, Entries 1 3 ). 当使用强碱时, 该反应会产生少量副产物(Table 1, Entries 7 9). 而使用 $\mathrm{Cs}_{2} \mathrm{CO}_{3} 、 \mathrm{KO}^{t} \mathrm{Bu} 、 \mathrm{NaO}^{t} \mathrm{Bu}$ 作为碱 时, 该反应能顺利进行且获得较高的反应收率(Table 1, Entries 4 6). 随后, 考察了溶剂对反应的影响. 结果表 明溶剂对该反应的影响很大，经过优化对比发现二甲苯 是最佳的反应溶剂(Table 1, Entries 4、10 14). 最

表 1 反应条件的优化 ${ }^{a}$

Table 1 Optimization of reaction conditions

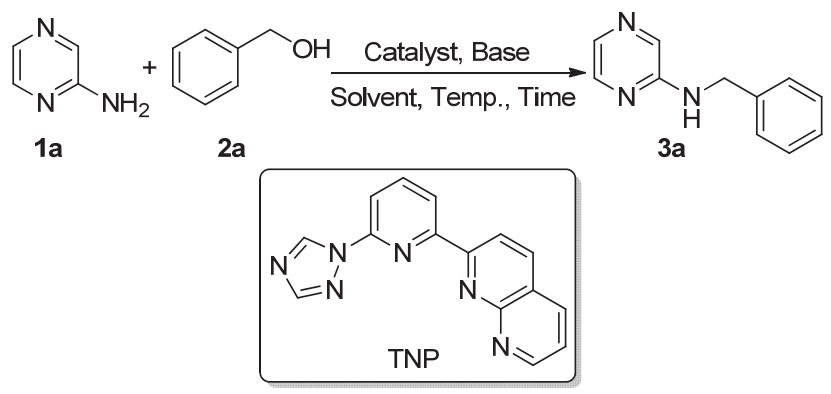

\begin{tabular}{ccccc}
\hline Entry & Base & Solvent & Temp. $/{ }^{\circ} \mathrm{C}$ & Yield $^{b} / \%$ \\
\hline 1 & $\mathrm{~K}_{2} \mathrm{CO}_{3}$ & Xylene & 140 & 24 \\
2 & $\mathrm{Na}_{2} \mathrm{CO}_{3}$ & Xylene & 140 & Trace \\
3 & $\mathrm{~K}_{3} \mathrm{PO}_{4}$ & Xylene & 140 & 33 \\
4 & $\mathrm{Cs}_{2} \mathrm{CO}_{3}$ & Xylene & $\mathbf{1 4 0}$ & $\mathbf{9 7}$ \\
5 & $\mathrm{KO}{ }^{t} \mathrm{Bu}$ & Xylene & 140 & 95 \\
6 & $\mathrm{NaO}{ }^{t} \mathrm{Bu}$ & Xylene & 140 & 91 \\
7 & $\mathrm{KOH}$ & Xylene & 140 & 89 \\
8 & $\mathrm{NaOH}$ & Xylene & 140 & 86 \\
9 & $\mathrm{CsOH} \mathrm{H}_{2} \mathrm{O}$ & Xylene & 140 & 75 \\
10 & $\mathrm{Cs}_{2} \mathrm{CO}_{3}$ & Toluene & 140 & 79 \\
11 & $\mathrm{Cs}_{2} \mathrm{CO}_{3}$ & $1,4-$ Dioxane & 140 & 30 \\
12 & $\mathrm{Cs}_{2} \mathrm{CO}_{3}$ & DMF & 140 & 61 \\
13 & $\mathrm{Cs}_{2} \mathrm{CO}_{3}$ & DMA & 140 & Trace \\
14 & $\mathrm{Cs}_{2} \mathrm{CO}_{3}$ & 1,2 -Ethanediol & 140 & Trace \\
15 & $\mathrm{Cs}_{2} \mathrm{CO}_{3}$ & Xylene & 120 & 76 \\
16 & $\mathrm{Cs}_{2} \mathrm{CO}_{3}$ & Xylene & 150 & 97 \\
17 & $\mathrm{Cs}_{2} \mathrm{CO}_{3}$ & Xylene & 140 & $85^{c}$ \\
18 & $\mathrm{Cs}_{2} \mathrm{CO}_{3}$ & Xylene & 140 & $97^{d}$ \\
19 & $\mathrm{Cs}_{2} \mathrm{CO}_{3}$ & Xylene & 140 & Trace \\
\hline
\end{tabular}

${ }^{a}$ Reagents and conditions: 1a $(1.0 \mathrm{mmol}), \mathbf{2 a}(1.2 \mathrm{mmol})$, catalyst $(15 \mathrm{mg}, \omega=$ $3 \%$ loading), solvent $(2.0 \mathrm{~mL})$, base $(0.75$ equiv. $), 8 \mathrm{~h} .{ }^{b}$ Yields of isolated product. ${ }^{c} 6 \mathrm{~h} .{ }^{d} 12 \mathrm{~h} .{ }^{e}$ Without catalyst. 
后，对反应时间及温度进行了优化，发现 $8 \mathrm{~h} 、 140{ }^{\circ} \mathrm{C}$ 为 该反应最优的反应时间和温度(Table 1, Entries 15 18).

\section{2 底物普适性的考察}

在最优反应条件的基础上, 对反应底物进行了官能 团普适性研究. 实验结果表明 TNP-Cu@rGo 对此反应 中大多数取代基的茮醇都有着良好的适应性, 取得较高 或者优秀的产率，具体见表 2 . 当苄醇苯环上带有供电 子基(如 $\mathrm{CH}_{3} 、 \mathrm{OCH}_{3}$ ) 该反应都能以优秀的收率得到相应 的目标产物(Table 2, 3b $\sim 3 \mathbf{g}$ ). 实验发现含吸电子基团 的苄醇也可以与氨基吡嗪顺利的发生反应, 都能够以中 等及以上收率得到目标产物(Table $2,3 \mathbf{h} \sim 3 \mathbf{r}$ ). 同时，实 验发现空间位阻对该反应的影响较小，当茮醇取代基为 双取代基团或䒺环时，该反应也能获得中等到优秀的产 率(Table 2, 3t $\sim 3 \mathbf{u}, 3 \mathbf{x}$ ). 当以噻吩甲醇为原料时, 实验 也可以顺利进行，目标产物 3w 产率达到 85\%.

基于该催化剂在氨基吡嗪烷基化反应中高活性的 表现，再将 TNP-Cu@rGo 应用至经典的苄醇与酮的借 氢反应中, 并对底物普适性进行了研究(表 3). 结果表
明，当苯环上取代基无论是吸电子基还是供电子基, TNP-Cu@rGo 都能使反应顺利地进行并获得较高的反 应收率，得到相应的目标产物. 另外对其他芳香杂环醇 也进行了探究，也表现出了较高的反应活性(Table 3, 6e). 因此, TNP-Cu@rGo 不仅在氨基吡嗪的烷基化反应 中有较高的催化活性, 对于茮醇与酮的反应中也具有较 好的催化性能.

\section{3 反应机理的探讨}

基于该非均相催化剂 TNP-Cu@ $\mathrm{rGO}$ 在氨基吡嗪与 茮醇的反应中表现出的优异催化活性, 为进一步了解该 催化剂及反应的历程, 接下来对反应的机理进行了探 索.

首先，用自由基捕获剂 2,2,6,6-四甲基-1-哌啶基氮 氧化合物(TEMPO)对此反应的机理类型进行鉴别. 结果 表明，当 TEMPO 存在条件下，氨基吡嗪与苯甲醇仍然 能够顺利进行，且以优秀的收率(91\%)得到相应的产物 3a, 由此可以初步排除该反应体系为自由基反应的可能 性(Scheme 2a).

表 2 底物的普适性考察 $a, b$

Table 2 Substrate scope of the reaction
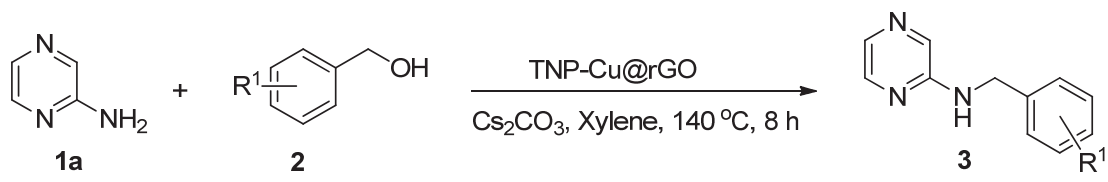<smiles>c1ccc(CNc2cnccn2)cc1</smiles>

3a, $97 \%$<smiles>Cc1ccccc1CNc1cnccn1</smiles>

3b, $83 \%$<smiles>Cc1cccc(CNc2cnccn2)c1</smiles>

$3 c, 85 \%$<smiles>Cc1ccc(CNc2cnccn2)cc1</smiles>

$3 d, 89 \%$<smiles>COc1ccccc1CNc1cnccn1</smiles>

$3 e, 88 \%$<smiles>COc1cccc(CNc2cnccn2)c1</smiles>

3f, $89 \%$<smiles>COc1ccc(CNc2cnccn2)cc1</smiles>

$3 g, 92 \%$

3h, $80 \%$

$\mathbf{3 i}, 84 \%$

$3 \mathbf{j}, 72 \%$

$3 \mathbf{k}, 78 \%$

3I, $81 \%$<smiles>FC(F)(F)c1ccc(CNc2cnccn2)cc1CNc1cnccc1CNc1cnccc1CNc1ccccc1C(F)(F)F</smiles>

$3 \mathrm{~m}, 80 \%$

3n, $83 \%$

3o, $87 \%$

$3 p, 82 \%$

$3 q, 84 \%$

3 r, $85 \%$<smiles>COc1cc(CNc2cnccn2)cc(OC)c1</smiles>

3s, $80 \%$

3t, $78 \%$

$3 u, 87 \%$

$3 v, 80 \%$

$3 w, 85 \%$

$3 x, 90 \%$

${ }^{a}$ Reagents and conditions: 1a $(1.0 \mathrm{mmol}), 2$ (1.2 mmol.), TNP-Cu@rGO (15 mg, $\omega=3 \%$ loading $)$, xylene $(2.0 \mathrm{~mL}), \mathrm{Cs}_{2} \mathrm{CO} 3(0.75$ equiv. $), 140{ }^{\circ} \mathrm{C}, 8 \mathrm{~h} .{ }^{b}$ Yields of isolated product. 
表 3 苄醇与酮反应的底物普适性考察 $a, b$

Table 3 Substrate expansion for the reaction of alcohols with ketones
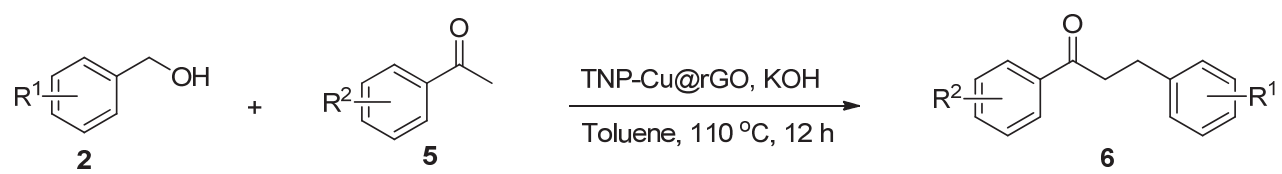

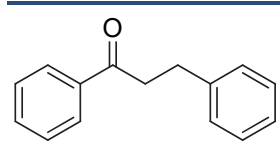

$6 a, 86 \%$<smiles>O=C(CCc1cccs1)c1ccccc1</smiles>

$6 e, 81 \%$

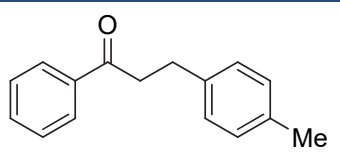

6b, $83 \%$

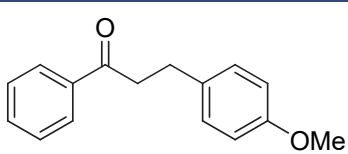

6c, $80 \%$<smiles>O=C(CCc1ccccc1Br)c1ccccc1</smiles>

$6 d, 88 \%$

${ }^{a}$ Reagents and conditions: 2 (1.1 mmol), 5 (1.0 mmol.), TNP-Cu@rGO (15 mg, $\omega=3 \%$ loading), toluene $(2.0 \mathrm{~mL}), \mathrm{KOH}\left(0.75\right.$ equiv.), $110{ }^{\circ} \mathrm{C}, 12 \mathrm{~h} .{ }^{b}$ Yields of isolated product.<smiles>O=C(CCc1ccccc1)c1ccc(Cl)cc1</smiles>

6f, $77 \%$<smiles>Cc1cccc(C(=O)CCc2ccccc2)c1</smiles>

$6 \mathrm{~g}, 79 \%$<smiles>COc1cccc(C(=O)CCc2ccccc2)c1</smiles>

6h, $82 \%$ (a)<smiles>Nc1cnccn1</smiles><smiles>c1ccc(CNc2cnccn2)cc1</smiles>

3a No radical scavenger: $97 \%$

(b)
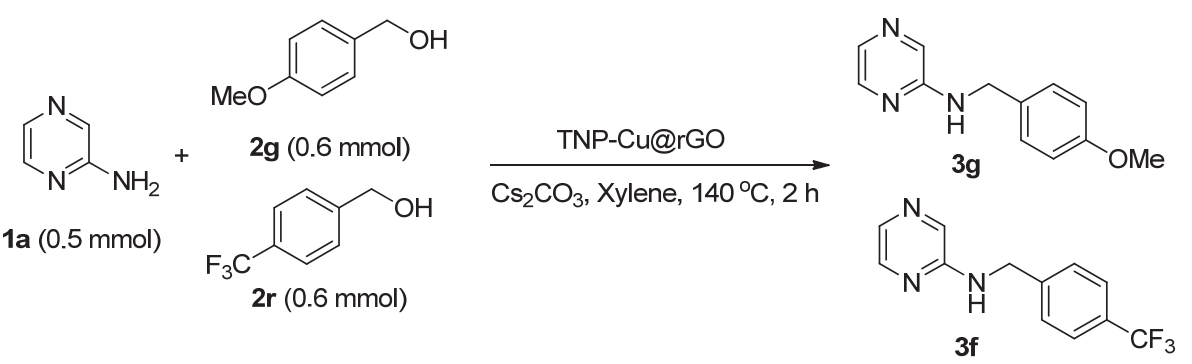

$3 g: 3 f=2.7: 1$

图式 2 控制实验

Scheme 2 Control experiments

随后，为阐明该反应取代基的电子效应对该反应的 影响，选择氨基吡嗪作为反应底物与不同取代基芐醇进 行反应 $(2 \mathrm{~g} 、 2 \mathrm{r})$. 反应 $2 \mathrm{~h}$ 后, 通过气相色谱 $(\mathrm{GC})$ 分析表 明, 两种芐醇所对应的预期产物 (3g 和 3f) 的物质的量比 为 $2.7: 1$. 结果表明此反应在初始阶段该体系会受到电 子云密度的影响, 当茮醇苯环上取代基被供电子基团取 代时，对该反应的发生更有利(Scheme 2b).

反应的动力学研究和中间体的捕捉对研究反应的 机理起着至关重要的作用. 随后, 尝试对氨基吡嗪与苯 甲醇反应的动力学进行探索. 在不同的反应时间下, 对 反应体系进行检测分析(图 1). 实验结果表明在初始阶 段苯甲醇脱氢生成一定量的苯甲醛中间体, 且苯甲醛的 形成和消耗在该反应过程中扮演着重要的作用. 同时还 发现反应进行 $8 \mathrm{~h}$, 产率达到最大. 即使再延长反应时 间，产物的产率也不会增加。

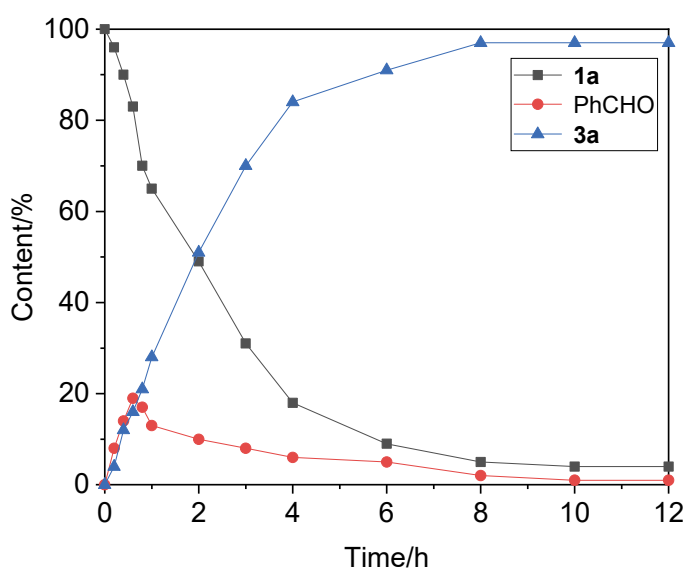

图 1 化合物 3a 合成过程中中间体的研究

Figure 1 Intermediate research for the synthesis of $\mathbf{3 a}$ 
此外, 还进行了三组中间体捕获和对照实验 (Scheme 3). 首先在最优的反应条件下, 当反应体系中 仅加入苯甲醇为反应物而未加入氨基吡嗪时, 结果表 明，该反应体系以 $14 \%$ 的收率得到苯甲醛(4a). 随后， 在同样的反应条件下, 让苯甲醛 $(\mathbf{4 a})$ 与氨基吡嗪反应 8 $h$, 最终得到亚胺中间体(4b). 最后, 将得到的亚胺中间 体中和苯甲醇反应, 仍然可以得到预期产物 3a. 这也从 实验上证明该反应是借氢反应的机理.

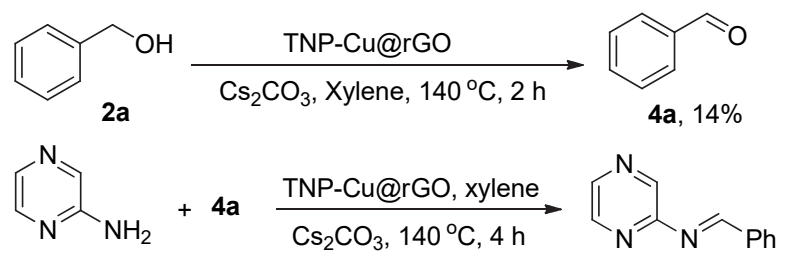

$1 \mathrm{a}$

4b, $96 \%$

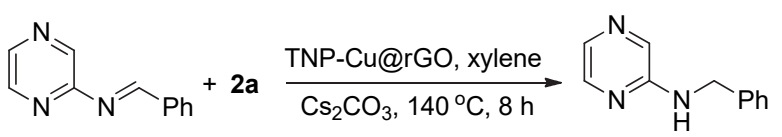

$4 \mathrm{~b}$

3a, $93 \%$

图式 3 中间体捕获实验

Scheme 3 Intermediate capture experiments

根据上面的实验和相关文献报道 ${ }^{[12]}$, 以合成预期 产物 3a 为例, 提出了该反应可能的机理如 Scheme 4 所 示. 该反应经过以下三个步骤: (i)在催化剂和碱的协同 作用下, 苯甲醇脱氢形成苯甲醛 4a. (ii) 苯甲醛与氨基吡 嗪发生缩合反应脱水生成亚胺中间体 $\mathbf{4 b}$. (iii) 第一步产 生的氢在催化剂的作用下原位还原亚胺中间体生成目 标产物 $3 \mathbf{a}$.

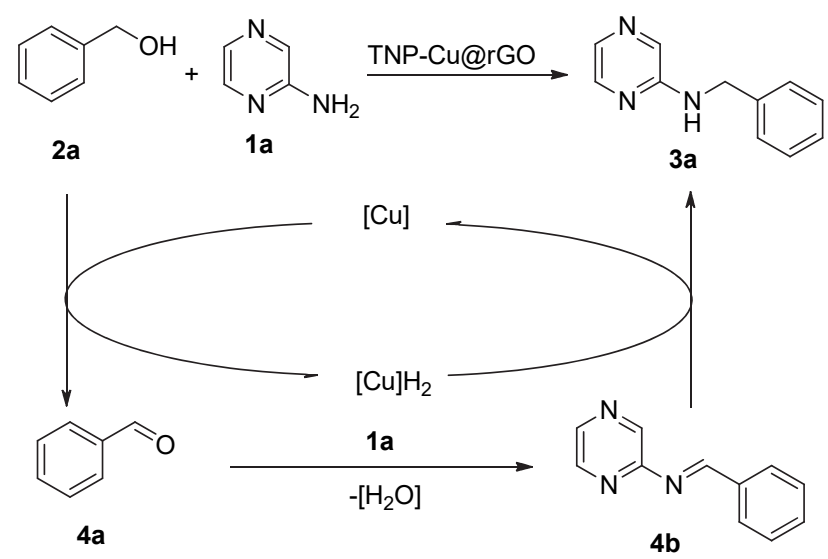

图式 4 合成 $3 a$ 的反应机理

Scheme 4 Reaction mechanism of the synthesis of $\mathbf{3 a}$

\section{4 催化剂 TNP-Cu@rGO 回收循环实验}

最后, 为了验证该非均相催化剂的循环利用性能, 对 TNP-Cu@rGO 的回收再利用实验进行了研究. 将反 应结束后的体系经过离心、用甲醇及蒸馏水多次洗涤、
干燥等步骤回收固体催化剂, 将所回收催化剂在最优条 件下再次投料进行反应(Scheme 5). 结果表明，随着反 应循环的使用次数的增多, 该催化剂的反应活性有略微 的降低, 预期产物 3a 仍然能够得到较高的反应收率.

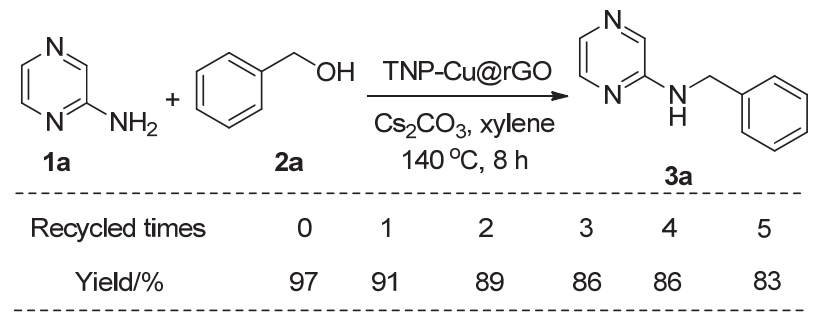

图式 5 催化剂 TNP-Cu@rGO 的回收实验

Scheme 5 Recycled experiments of TNP-Cu@rGO catalyst

\section{2 结论}

综上所述，本文利用非均相催化剂 TNP-Cu@rGO 实现了廉价金属铜催化氨基吡嗪与苄醇以及醇与酮的 借氢反应. 氨基吡嗪与芐醇的借氢反应底物普适性实验 表明该催化剂对不同官能团取代的茮醇均表现出了较 强的催化活性，取得了 $72 \%$ \% 97\%的产率. 通过控制实 验以及对中间体的捕获实验推测出了该反应的反应历 程. 同时, 对该非均相催化剂进行了回收再利用实验. 该催化体系提供了一种有效合成烷基吡嗪化合物的方 法并实现了可循环再利用, 这符合可持续发展理念也为 今后合成取代吡嗪衍生物提供了一条切实可行的路线.

\section{3 实验部分}

\section{1 仪器与试剂}

所有得到的产物均采用 ${ }^{1} \mathrm{H}-\mathrm{NMR} 、{ }^{13} \mathrm{C}-\mathrm{NMR}$ 和 ${ }^{19} \mathrm{~F}$ NMR 进行表征，并以四甲基硅烷(TMS)为内标 $(\delta 0)$, 参 考 DMSO- $d_{6}\left({ }^{1} \mathrm{H}\right.$ 为 $\delta 2.50,{ }^{13} \mathrm{C}$ 为 $\left.\delta 39.5\right)$ 或 $\mathrm{CDCl}_{3}\left({ }^{1} \mathrm{H}\right.$ 为 $\delta 7.26,{ }^{13} \mathrm{C}$ 为 $\delta$ 77.1). ${ }^{1} \mathrm{H}-\mathrm{NMR},{ }^{13} \mathrm{C}-\mathrm{NMR}$ 和 ${ }^{19} \mathrm{~F}-\mathrm{NMR}$ 分 别在 Bruker Advance III HD $400 \mathrm{MHz} 、 101 \mathrm{MHz}$ 和 376 $\mathrm{Hz}$ 谱仪上获得. 采用市售 100 400 目硅胶板(SGF254) 进行薄层分析. 在 $230 \sim 430$ 目硅胶上进行柱层析分离. 高分辨质谱(HRMS)在 LTQ-FTUHRA 质谱仪上记录. 所 有试剂均由商业供应商提供, 使用时无需进一步净化.

\section{2 实验方法}

3.2.1 TNP-Cu@rGO 催化的氨基吡嗪与華醇偶联的 实验方法

在 $25 \mathrm{~mL}$ 干燥反应管中, 依次加入苯甲醇(2a)(130 $\mathrm{mg}, 1.2 \mathrm{mmol}) 、$ 氨基吡嗪(1a) $(95 \mathrm{mg}, 1.0 \mathrm{mmol}) 、$ 催化 剂 TNP-Cu@rGO (15 mg, 3 wt \%)、 $\mathrm{Cs}_{2} \mathrm{CO}_{3}(244 \mathrm{mg}, 0.75$ $\mathrm{mmol})$ 、二甲苯 $(2.0 \mathrm{~mL})$ 于 $140{ }^{\circ} \mathrm{C}$ 油浴锅中摚拌反应 $8 \mathrm{~h}$, 反应过程中用薄层色谱(TLC)追踪反应进度, 反应结束 
后, 待其冷却至室温, 将反应物中加入水淬灭并加入乙 酸乙酯 $(5 \mathrm{~mL} \times 3)$ 萃取, 合并有机相并用无水 $\mathrm{MgSO}_{4}$ 干 燥, 减压浓缩得到粗产品并采用柱层析纯化 [ $V$ (石油 醚) $: V($ 乙酸乙酯 $)=5: 1$ ], 得到 $N$-芐基吡嗪-2-胺(3a): 淡黄色固体，180 mg, 产率 97\%. m.p. $65 \sim 66{ }^{\circ} \mathrm{C}$ (Ref. $\left.{ }^{[13 a]} 66 \sim 67{ }^{\circ} \mathrm{C}\right) ;{ }^{1} \mathrm{H}$ NMR (400 MHz, $\left.\mathrm{CDCl}_{3}\right) \delta: 7.78$ (dd, $J=2.8,1.5 \mathrm{~Hz}, 1 \mathrm{H}), 7.74 \sim 7.67(\mathrm{~m}, 1 \mathrm{H}), 7.61(\mathrm{~d}, J=$ $2.9 \mathrm{~Hz}, 1 \mathrm{H}), 7.19$ (d, $J=4.5 \mathrm{~Hz}, 4 \mathrm{H}), 7.14$ (q, $J=4.1 \mathrm{~Hz}$, $1 \mathrm{H}), 5.54(\mathrm{~d}, J=5.6 \mathrm{~Hz}, 1 \mathrm{H}), 4.39(\mathrm{~d}, J=5.7 \mathrm{~Hz}, 2 \mathrm{H}) ;{ }^{13} \mathrm{C}$ NMR $\left(101 \mathrm{MHz}, \mathrm{CDCl}_{3}\right) \delta$ : 153.54, 140.87, 137.51, $131.61,131.05,127.62,126.50,126.37,44.32$; HRMS calcd for $\mathrm{C}_{11} \mathrm{H}_{12} \mathrm{~N}_{3}[\mathrm{M}+\mathrm{H}]^{+}$186.1031, found 186.1031 .

$\mathrm{N}$-(2-甲基苄基吡嗪)-2-胺(3b): 黄色固体, $165 \mathrm{mg}$, 产率 83\%. m.p. $67 \sim 68{ }^{\circ} \mathrm{C} ;{ }^{1} \mathrm{H}$ NMR $\left(400 \mathrm{MHz}, \mathrm{CDCl}_{3}\right)$ $\delta: 7.80$ (dd, $J=2.8,1.5 \mathrm{~Hz}, 1 \mathrm{H}), 7.71(\mathrm{~d}, J=1.5 \mathrm{~Hz}, 1 \mathrm{H})$, 7.63 (d, $J=2.8 \mathrm{~Hz}, 1 \mathrm{H}), 7.17$ (d, $J=7.3 \mathrm{~Hz}, 1 \mathrm{H}), 7.07$ (td, $J=6.5,4.3 \mathrm{~Hz}, 3 \mathrm{H}), 5.21(\mathrm{t}, J=5.4 \mathrm{~Hz}, 1 \mathrm{H}), 4.38(\mathrm{~d}, J=$ $5.3 \mathrm{~Hz}, 2 \mathrm{H}), 2.23(\mathrm{~s}, 3 \mathrm{H}) ;{ }^{13} \mathrm{C} \mathrm{NMR}\left(101 \mathrm{MHz}, \mathrm{CDCl}_{3}\right) \delta$ : $154.57,141.98,136.50,136.16,132.82,132.14,130.60$, 128.31, 127.77, 126.25, 43.69, 19.03; HRMS calcd for $\mathrm{C}_{12} \mathrm{H}_{14} \mathrm{~N}_{3}[\mathrm{M}+\mathrm{H}]^{+}$200.1188, found 200.1186.

$\mathrm{N}$-(3-甲基苄基吡嗪)-2-胺(3c)：黄色油状液体，169 $\mathrm{mg}$, 产率 85\%. ${ }^{1} \mathrm{H}$ NMR $\left(400 \mathrm{MHz}, \mathrm{CDCl}_{3}\right) \delta: 7.89(\mathrm{dd}$, $J=2.8,1.5 \mathrm{~Hz}, 1 \mathrm{H}), 7.81(\mathrm{~s}, 1 \mathrm{H}), 7.71(\mathrm{~d}, J=2.8 \mathrm{~Hz}, 1 \mathrm{H})$, 7.19 (t, $J=7.5 \mathrm{~Hz}, 1 \mathrm{H}), 7.10(\mathrm{~d}, J=9.1 \mathrm{~Hz}, 2 \mathrm{H}), 7.05$ (d, $J=7.5 \mathrm{~Hz}, 1 \mathrm{H}), 5.63(\mathrm{~s}, 1 \mathrm{H}), 4.45(\mathrm{~d}, J=5.5 \mathrm{~Hz}, 2 \mathrm{H}), 2.29$ (s, 3H); ${ }^{13} \mathrm{C}$ NMR $\left(101 \mathrm{MHz}, \mathrm{CDCl}_{3}\right) \delta: 154.69,141.98$, $138.53,138.39,132.66,132.16,128.65,128.37,128.24$, 124.67, 45.44, 21.43; HRMS calcd for $\mathrm{C}_{12} \mathrm{H}_{14} \mathrm{~N}_{3}[\mathrm{M}+\mathrm{H}]^{+}$ 200.1188 , found 200.1187 .

$\mathrm{N}$-(4-甲基苄基吡嗪)-2-胺(3d)：黄色固体，177 mg, 产率 89\%. m.p. $77 \sim 78{ }^{\circ} \mathrm{C}$ (Ref. ${ }^{[13 a]} 78 \sim 80{ }^{\circ} \mathrm{C}$ ); ${ }^{1} \mathrm{H}$ NMR $\left(400 \mathrm{MHz}, \mathrm{CDCl}_{3}\right) \delta: 7.93 \sim 7.59(\mathrm{~m}, 3 \mathrm{H}), 7.13(\mathrm{~d}$, $J=7.7 \mathrm{~Hz}, 2 \mathrm{H}), 7.04(\mathrm{~d}, J=7.7 \mathrm{~Hz}, 2 \mathrm{H}), 5.22$ (t, $J=5.9$ $\mathrm{Hz}, 1 \mathrm{H}), 4.38(\mathrm{~d}, J=5.6 \mathrm{~Hz}, 2 \mathrm{H}), 2.23(\mathrm{~s}, 3 \mathrm{H}) ;{ }^{13} \mathrm{C} \mathrm{NMR}$ $\left(101 \mathrm{MHz}, \mathrm{CDCl}_{3}\right) \delta: 154.60,142.01,137.20,135.47$, 132.85, 132.11, 129.43, 127.62, 45.31, 21.13; HRMS calcd for $\mathrm{C}_{12} \mathrm{H}_{14} \mathrm{~N}_{3}[\mathrm{M}+\mathrm{H}]^{+} 200.1188$, found 200.1186 .

$\mathrm{N}$-(2-甲氧基苠基吡嗪)-2-胺(3e): 黄色固体, 189 $\mathrm{mg}$, 产率 88\%. m.p. $70 \sim 71{ }^{\circ} \mathrm{C} ;{ }^{1} \mathrm{H}$ NMR $(400 \mathrm{MHz}$, $\left.\mathrm{CDCl}_{3}\right) \delta: 7.92 \sim 7.82(\mathrm{~m}, 1 \mathrm{H}), 7.77(\mathrm{~s}, 1 \mathrm{H}), 7.64(\mathrm{~s}, 1 \mathrm{H})$, $7.27 \sim 7.09(\mathrm{~m}, 2 \mathrm{H}), 6.86 \sim 6.69(\mathrm{~m}, 2 \mathrm{H}), 5.28(\mathrm{~s}, 1 \mathrm{H})$, 4.44 (d, $J=5.9 \mathrm{~Hz}, 2 \mathrm{H}), 3.73$ (s, 3H); ${ }^{13} \mathrm{C}$ NMR $(101 \mathrm{MHz}$, $\left.\mathrm{CDCl}_{3}\right) \delta: 157.57,154.79,141.96,132.57,132.27,129.26$, $128.79,126.48,120.56,110.37,55.30,41.06$; HRMS calcd for $\mathrm{C}_{12} \mathrm{H}_{14} \mathrm{~N}_{3} \mathrm{O}[\mathrm{M}+\mathrm{H}]^{+}$216.1137, found 216.1136. $N$-(3-甲氧基芐基吡嗪)-2-胺 (3f $)^{[13 b]}$ : 淡黄色液体, $191 \mathrm{mg}$, 产率 89\%. ${ }^{1} \mathrm{H}$ NMR (400 MHz, $\mathrm{CDCl}_{3}$ ) $\delta: 7.92$ (dd, $J=2.8,1.5 \mathrm{~Hz}, 1 \mathrm{H}), 7.83(\mathrm{~d}, J=1.5 \mathrm{~Hz}, 1 \mathrm{H}), 7.74(\mathrm{~d}$, $J=2.8 \mathrm{~Hz}, 1 \mathrm{H}), 7.22(\mathrm{t}, J=7.9 \mathrm{~Hz}, 1 \mathrm{H}), 6.93 \sim 6.84(\mathrm{~m}$, $2 \mathrm{H}), 6.79$ (ddd, $J=8.2,2.6,0.9 \mathrm{~Hz}, 1 \mathrm{H}), 5.61(\mathrm{~s}, 1 \mathrm{H}), 4.48$ (d, $J=5.7 \mathrm{~Hz}, 2 \mathrm{H}), 3.73$ (s, 3H); ${ }^{13} \mathrm{C}$ NMR $(101 \mathrm{MHz}$, $\left.\mathrm{CDCl}_{3}\right) \delta: 159.89,154.61,141.96,140.27,132.75,132.18$, 129.76, 119.78, 113.21, 112.75, 55.18, 45.35; HRMS calcd for $\mathrm{C}_{12} \mathrm{H}_{14} \mathrm{~N}_{3} \mathrm{O}[\mathrm{M}+\mathrm{H}]^{+}$216.1137, found 216.1138.

$\mathrm{N}$-(4-甲氧基苄基吡嗪)-2-胺(3g): 黄色固体, 198 mg, 产率 92\%. m.p. 96 97 ${ }^{\circ} \mathrm{C}$ (Ref. ${ }^{[13 c]} 97 \sim 99{ }^{\circ} \mathrm{C}$ ); ${ }^{1} \mathrm{H}$ NMR (400 MHz, $\left.\mathrm{CDCl}_{3}\right) \delta: 7.85(\mathrm{dd}, J=2.8,1.5 \mathrm{~Hz}, 1 \mathrm{H})$, $7.77 \sim 7.72(\mathrm{~m}, 1 \mathrm{H}), 7.67(\mathrm{~d}, J=2.8 \mathrm{~Hz}, 1 \mathrm{H}), 7.20 \sim 7.12$ (m, 2H), 6.83 6.68 (m, 2H), $5.21(\mathrm{~s}, 1 \mathrm{H}), 4.36(\mathrm{~d}, J=5.4$ $\mathrm{Hz}, 2 \mathrm{H}), 3.68(\mathrm{~s}, 3 \mathrm{H}) ;{ }^{13} \mathrm{C}$ NMR (101 MHz, $\left.\mathrm{CDCl}_{3}\right) \delta$ : $159.03,154.54,141.97,132.80,132.15,130.53,128.96$, 114.12, 55.30, 45.00; HRMS calcd for $\mathrm{C}_{12} \mathrm{H}_{14} \mathrm{~N}_{3} \mathrm{O}$ [M+ $\mathrm{H}]^{+}$216.1137, found 216.1137 .

$\mathrm{N}$-(3-氟苄基吡嗪)-2-胺(3h): 黄色液体, $162 \mathrm{mg}$, 产 率 80\%. ${ }^{1} \mathrm{H}$ NMR $\left(400 \mathrm{MHz}, \mathrm{CDCl}_{3}\right) \delta: 7.87(\mathrm{~d}, J=1.3$ Hz, 1H), 7.78 (s, 1H), 7.70 (d, $J=2.8 \mathrm{~Hz}, 1 \mathrm{H}), 7.18$ (td, $J=7.9,5.8 \mathrm{~Hz}, 1 \mathrm{H}), 7.01(\mathrm{~d}, J=7.6 \mathrm{~Hz}, 1 \mathrm{H}), 6.94(\mathrm{dt}, J=$ 9.7, $2.1 \mathrm{~Hz}, 1 \mathrm{H}), 6.85$ (td, $J=8.4,2.6 \mathrm{~Hz}, 1 \mathrm{H}), 5.38$ (s, $1 \mathrm{H}), 4.46(\mathrm{~d}, J=5.8 \mathrm{~Hz}, 2 \mathrm{H}) ;{ }^{13} \mathrm{C} \mathrm{NMR}\left(101 \mathrm{MHz}, \mathrm{CDCl}_{3}\right)$ $\delta: 161.99$ (d, $J=246.2 \mathrm{~Hz}), 153.36,140.94,140.33$ (d, $J=$ $6.9 \mathrm{~Hz}), 132.06,131.15,129.18(\mathrm{~d}, J=8.3 \mathrm{~Hz}), 121.89$ (d, $J=2.8 \mathrm{~Hz}), 113.35,113.14$ (d, $J=1.1 \mathrm{~Hz}), 43.76$ (d, $J=$ $1.9 \mathrm{~Hz}$ ); ${ }^{19} \mathrm{~F}$ NMR (376 MHz, $\left.\mathrm{CDCl}_{3}\right) \delta:-112.67$; HRMS calcd for $\mathrm{C}_{11} \mathrm{H}_{11} \mathrm{~N}_{3} \mathrm{~F}[\mathrm{M}+\mathrm{H}]^{+}$204.0937, found 204.0938.

$\mathrm{N}$-(4-氟苄基吡嗪)-2-胺(3i): 黄色固体, $170 \mathrm{mg}$, 产 率 84\%. m.p. $79 \sim 80{ }^{\circ} \mathrm{C} ;{ }^{1} \mathrm{H}$ NMR $\left(400 \mathrm{MHz}, \mathrm{CDCl}_{3}\right) \delta$ : $7.86(\mathrm{dd}, J=2.8,1.5 \mathrm{~Hz}, 1 \mathrm{H}), 7.81 \sim 7.72(\mathrm{~m}, 1 \mathrm{H}), 7.68(\mathrm{~d}$, $J=2.8 \mathrm{~Hz}, 1 \mathrm{H}), 7.28 \sim 7.11(\mathrm{~m}, 2 \mathrm{H}), 6.99 \sim 6.84(\mathrm{~m}, 2 \mathrm{H})$, $5.34(\mathrm{t}, J=5.7 \mathrm{~Hz}, 1 \mathrm{H}), 4.41(\mathrm{~d}, J=5.5 \mathrm{~Hz}, 2 \mathrm{H}) ;{ }^{13} \mathrm{C} \mathrm{NMR}$ $\left(101 \mathrm{MHz}, \mathrm{CDCl}_{3}\right) \delta: 162.15(\mathrm{~d}, J=245.5 \mathrm{~Hz}), 154.45$, $141.98,134.35$ (d, $J=3.1 \mathrm{~Hz}), 132.97,132.18,129.21$ (d, $J=8.0 \mathrm{~Hz}), 115.53(\mathrm{~d}, J=21.4 \mathrm{~Hz}), 44.70 ;{ }^{19} \mathrm{~F}$ NMR $(376$ $\mathrm{MHz}, \mathrm{CDCl}_{3}$ ) $\delta$ : -115.03 ; HRMS calcd for $\mathrm{C}_{11} \mathrm{H}_{11} \mathrm{~N}_{3} \mathrm{~F}$ $[\mathrm{M}+\mathrm{H}]^{+}$204.0937, found 204.0937.

$N$-(2-氯苄基吡嗪)-2-胺 $(\mathbf{3 j})^{[13 \mathrm{~d}]}$ : 黄绿色固体, 158 $\mathrm{mg}$, 产率 $72 \%$. m.p. $71 \sim 72{ }^{\circ} \mathrm{C}$; ${ }^{1} \mathrm{H}$ NMR $(400 \mathrm{MHz}$, $\left.\mathrm{CDCl}_{3}\right) \delta: 7.96 \sim 7.94(\mathrm{~m}, 1 \mathrm{H}), 7.86(\mathrm{~d}, J=1.6 \mathrm{~Hz}, 1 \mathrm{H})$, $7.77(\mathrm{~d}, J=2.8 \mathrm{~Hz}, 1 \mathrm{H}), 7.38(\mathrm{dd}, J=5.7,2.2 \mathrm{~Hz}, 1 \mathrm{H})$, $7.32(\mathrm{~d}, J=4.6 \mathrm{~Hz}, 1 \mathrm{H}), 7.19(\mathrm{dd}, J=5.8,3.5 \mathrm{~Hz}, 2 \mathrm{H})$, 
$5.50(\mathrm{~s}, 1 \mathrm{H}), 4.64(\mathrm{~d}, J=6.1 \mathrm{~Hz}, 2 \mathrm{H}) ;{ }^{13} \mathrm{C}$ NMR $(101 \mathrm{MHz}$, $\left.\mathrm{CDCl}_{3}\right) \delta: 154.43,141.96,135.96,133.59,133.02,132.30$, 129.64, 128.79, 127.60, 126.97, 43.19.; HRMS calcd for $\mathrm{C}_{11} \mathrm{H}_{11} \mathrm{~N}_{3} \mathrm{Cl}[\mathrm{M}+\mathrm{H}]^{+}$220.0642, found 220.0643 .

$\mathrm{N}$-(3-氯芐基吡嗪)-2-胺(3k $)^{[13 \mathrm{~d}]}$ : 黄色液体, $171 \mathrm{mg}$, 产率 78\%. ${ }^{1} \mathrm{H}$ NMR $\left(400 \mathrm{MHz}, \mathrm{CDCl}_{3}\right) \delta: 7.84(\mathrm{dd}, J=$ $2.8,1.5 \mathrm{~Hz}, 1 \mathrm{H}), 7.75(\mathrm{~s}, 1 \mathrm{H}), 7.65(\mathrm{~d}, J=2.7 \mathrm{~Hz}, 1 \mathrm{H})$, $7.20(\mathrm{~d}, J=2.2 \mathrm{~Hz}, 1 \mathrm{H}), 7.10(\mathrm{qd}, J=5.3,2.6 \mathrm{~Hz}, 3 \mathrm{H})$, $5.58(\mathrm{~s}, 1 \mathrm{H}), 4.40(\mathrm{~d}, J=5.8 \mathrm{~Hz}, 2 \mathrm{H}) ;{ }^{13} \mathrm{C}$ NMR $(101 \mathrm{MHz}$, $\left.\mathrm{CDCl}_{3}\right) \delta: 154.43,141.99,140.89,134.49,132.96,132.25$, 129.97, 127.54, 127.52, 125.58, 44.72; HRMS calcd for $\mathrm{C}_{11} \mathrm{H}_{11} \mathrm{~N}_{3} \mathrm{Cl}[\mathrm{M}+\mathrm{H}]^{+}$220.0642, found 220.0644 .

$N$-(4-氯芐基吡嗪)-2-胺(3l): 黄色固体, $178 \mathrm{mg}$, 产 率 $81 \%$. m.p. $80 \sim 81{ }^{\circ} \mathrm{C}$ (Ref. ${ }^{[13 a]} 79 \sim 80{ }^{\circ} \mathrm{C}$ ); ${ }^{1} \mathrm{H}$ NMR $\left(400 \mathrm{MHz}, \mathrm{CDCl}_{3}\right) \delta: 7.96(\mathrm{dd}, J=2.8,1.5 \mathrm{~Hz}, 1 \mathrm{H}), 7.85$ (s, 1H), $7.79(\mathrm{~d}, J=2.8 \mathrm{~Hz}, 1 \mathrm{H}), 7.34 \sim 7.19(\mathrm{~m}, 4 \mathrm{H}), 5.37$ $(\mathrm{t}, J=6.0 \mathrm{~Hz}, 1 \mathrm{H}), 4.51(\mathrm{~d}, J=5.7 \mathrm{~Hz}, 2 \mathrm{H}) ;{ }^{13} \mathrm{C}$ NMR $(101$ $\left.\mathrm{MHz}, \mathrm{CDCl}_{3}\right) \delta: 154.38,141.99,137.17,133.18,133.13$, $132.19,128.87,128.83$, 44.72; HRMS calcd for $\mathrm{C}_{11} \mathrm{H}_{11} \mathrm{~N}_{3} \mathrm{Cl}[\mathrm{M}+\mathrm{H}]^{+}$220.0642, found 220.0642 .

$N$-(3-澳芳基吡嗪)-2-胺 $(3 \mathbf{m})$ : 黄色固体, $211 \mathrm{mg}$, 产 率 80\%. m.p. $60 \sim 61{ }^{\circ} \mathrm{C} ;{ }^{1} \mathrm{H}$ NMR $\left(400 \mathrm{MHz}, \mathrm{CDCl}_{3}\right) \delta$ : $8.09 \sim 7.72(\mathrm{~m}, 3 \mathrm{H}), 7.48(\mathrm{t}, J=1.9 \mathrm{~Hz}, 1 \mathrm{H}), 7.39$ (dt, $J=$ $7.9,1.6 \mathrm{~Hz}, 1 \mathrm{H}), 7.25(\mathrm{dt}, J=7.7,1.4 \mathrm{~Hz}, 1 \mathrm{H}), 7.18(\mathrm{t}, J=$ $7.8 \mathrm{~Hz}, 1 \mathrm{H}), 5.36(\mathrm{~s}, 1 \mathrm{H}), 4.53(\mathrm{~d}, J=5.6 \mathrm{~Hz}, 2 \mathrm{H}) ;{ }^{13} \mathrm{C}$ NMR $\left(101 \mathrm{MHz}, \mathrm{CDCl}_{3}\right) \delta$ : 154.32, 142.01, 141.10, $133.20,132.25,130.54,130.47,130.29,126.07,122.79$, 44.75; HRMS calcd for $\mathrm{C}_{11} \mathrm{H}_{11} \mathrm{~N}_{3} \mathrm{Br}[\mathrm{M}+\mathrm{H}]^{+}$264.0136, found 264.0137.

$\mathrm{N}$-(4-溴苄基吡嗪)-2-胺(3n): 黄色固体, $219 \mathrm{mg}$, 产 率 83\%. m.p. $112 \sim 113{ }^{\circ} \mathrm{C} ;{ }^{1} \mathrm{H}$ NMR $\left(400 \mathrm{MHz}, \mathrm{CDCl}_{3}\right)$ $\delta: 8.06 \sim 7.93(\mathrm{~m}, 1 \mathrm{H}), 7.93 \sim 7.72(\mathrm{~m}, 2 \mathrm{H}), 7.58 \sim 7.38$ (m, 2H), $7.27 \sim 7.11(\mathrm{~m}, 2 \mathrm{H}), 5.30(\mathrm{~s}, 1 \mathrm{H}), 4.50(\mathrm{~d}, J=5.5$ $\mathrm{Hz}, 2 \mathrm{H}) ;{ }^{13} \mathrm{C}$ NMR (101 MHz, $\left.\mathrm{CDCl}_{3}\right) \delta: 154.34,141.99$, $137.69,133.20,132.19,131.79,129.22,121.28,44.78$; HRMS calcd for $\mathrm{C}_{11} \mathrm{H}_{11} \mathrm{~N}_{3} \mathrm{Br}[\mathrm{M}+\mathrm{H}]^{+}$264.0136, found 264.0135 .

$N$-(4-碘苄基吡嗪)-2-胺(3o): 黄绿色固体, $270 \mathrm{mg}$, 产率 87\%. m.p. $126 \sim 127{ }^{\circ} \mathrm{C}$; ${ }^{1} \mathrm{H}$ NMR $(400 \mathrm{MHz}$, $\left.\mathrm{CDCl}_{3}\right) \delta: 7.90(\mathrm{dd}, J=2.8,1.5 \mathrm{~Hz}, 1 \mathrm{H}), 7.79(\mathrm{~s}, 1 \mathrm{H}), 7.74$ $(\mathrm{d}, J=2.8 \mathrm{~Hz}, 1 \mathrm{H}), 7.64 \sim 7.47(\mathrm{~m}, 2 \mathrm{H}), 7.08 \sim 6.94(\mathrm{~m}$, 2H), 5.07 (s, 1H), 4.43 (d, $J=5.2 \mathrm{~Hz}, 2 \mathrm{H}) ;{ }^{13} \mathrm{C}$ NMR $(101$ $\left.\mathrm{MHz}, \mathrm{CDCl}_{3}\right) \delta: 154.29,141.99,138.34,137.78,133.30$, 132.18, 129.47, 92.81, 44.91; HRMS calcd for $\mathrm{C}_{11} \mathrm{H}_{11} \mathrm{~N}_{3} \mathrm{I}$ $[\mathrm{M}+\mathrm{H}]^{+}$311.9998, found 311.9999.
$N$-(2-(三氟甲基)苄基吡嗪)-2-胺(3p)：黄色固体， $207 \mathrm{mg}$, 产率 82\%. m.p. $118 \sim 119{ }^{\circ} \mathrm{C} ;{ }^{1} \mathrm{H}$ NMR (400 $\left.\mathrm{MHz}, \mathrm{CDCl}_{3}\right) \delta: 8.04 \sim 7.95(\mathrm{~m}, 1 \mathrm{H}), 7.87(\mathrm{~s}, 1 \mathrm{H}), 7.81(\mathrm{~d}$, $J=2.8 \mathrm{~Hz}, 1 \mathrm{H}), 7.67(\mathrm{~d}, J=7.8 \mathrm{~Hz}, 1 \mathrm{H}), 7.58(\mathrm{~d}, J=7.7$ $\mathrm{Hz}, 1 \mathrm{H}), 7.49$ (t, $J=7.5 \mathrm{~Hz}, 1 \mathrm{H}), 7.37$ (t, $J=7.6 \mathrm{~Hz}, 1 \mathrm{H})$, $5.25(\mathrm{~s}, 1 \mathrm{H}), 4.79(\mathrm{~d}, J=5.9 \mathrm{~Hz}, 2 \mathrm{H}) ;{ }^{13} \mathrm{C}$ NMR $(101 \mathrm{MHz}$, $\left.\mathrm{CDCl}_{3}\right) \delta: 154.27,141.95,137.20,133.28,132.22,132.20$, $129.50,128.58$ (q, $J=272.1 \mathrm{~Hz}), 128.56(\mathrm{q}, J=30.1 \mathrm{~Hz})$, 127.47, 126.17 (q, $J=5.7 \mathrm{~Hz}), 41.78(\mathrm{q}, J=2.7 \mathrm{~Hz}) ;{ }^{19} \mathrm{~F}$ NMR $\left(376 \mathrm{MHz}, \mathrm{CDCl}_{3}\right) \delta$ : -59.81 ; HRMS calcd for $\mathrm{C}_{12} \mathrm{H}_{11} \mathrm{~N}_{3} \mathrm{~F}_{3}[\mathrm{M}+\mathrm{H}]^{+}$254.0905, found 254.0904.

$N$-(3-(三氟甲基)芐基吡嗪)-2-胺 (3q)：黄色液体， $213 \mathrm{mg}$, 产率 84\%. ${ }^{1} \mathrm{H}$ NMR (400 MHz, $\left.\mathrm{CDCl}_{3}\right) \delta: 7.87$ (s, 1H), 7.79 (s, 1H), 7.69 (d, $J=2.7 \mathrm{~Hz}, 1 \mathrm{H}), 7.50(\mathrm{~s}, 1 \mathrm{H})$, $7.46 \sim 7.38(\mathrm{~m}, 2 \mathrm{H}), 7.37 \sim 7.28(\mathrm{~m}, 1 \mathrm{H}), 5.46(\mathrm{~s}, 1 \mathrm{H})$, $4.52(\mathrm{~d}, J=5.7 \mathrm{~Hz}, 2 \mathrm{H}) ;{ }^{13} \mathrm{C}$ NMR $\left(101 \mathrm{MHz}, \mathrm{CDCl}_{3}\right) \delta$ : $154.37,141.98,139.84,133.17,132.30,131.44$ (q, $J=32.0$ $\mathrm{Hz}), 130.83,130.82,129.15,128.12(\mathrm{q}, J=271.5 \mathrm{~Hz})$, 124.26 (q, $J=4.4 \mathrm{~Hz}), 44.83 ;{ }^{19} \mathrm{~F} \mathrm{NMR}\left(376 \mathrm{MHz}, \mathrm{CDCl}_{3}\right)$ $\delta$ : -62.57 ; HRMS calcd for $\mathrm{C}_{12} \mathrm{H}_{11} \mathrm{~N}_{3} \mathrm{~F}_{3}[\mathrm{M}+\mathrm{H}]^{+}$ 254.0905, found 254.0903.

$N$-(4-(三氟甲基)芐基吡嗪)-2-胺(3r): 黄色固体, 215 $\mathrm{mg}$, 产率 85\%. m.p. $81 \sim 82{ }^{\circ} \mathrm{C}$; ${ }^{1} \mathrm{H}$ NMR $(400 \mathrm{MHz}$, $\left.\mathrm{CDCl}_{3}\right) \delta: 7.98(\mathrm{dd}, J=2.8,1.5 \mathrm{~Hz}, 1 \mathrm{H}), 7.89$ (d, $J=1.5$ $\mathrm{Hz}, 1 \mathrm{H}), 7.81(\mathrm{~d}, J=2.8 \mathrm{~Hz}, 1 \mathrm{H}), 7.58(\mathrm{~d}, J=8.1 \mathrm{~Hz}, 2 \mathrm{H})$, $7.45(\mathrm{~d}, J=8.0 \mathrm{~Hz}, 2 \mathrm{H}), 5.39$ (s, 1H), $4.63(\mathrm{~d}, J=5.7 \mathrm{~Hz}$, $2 \mathrm{H}) ;{ }^{13} \mathrm{C}$ NMR $\left(101 \mathrm{MHz}, \mathrm{CDCl}_{3}\right) \delta: 154.31,142.89$, $142.00,133.33,132.26,128.18$ (q, $J=32.5 \mathrm{~Hz}), 129.66$ (q, $J=271.5 \mathrm{~Hz}), 127.61,125.63(\mathrm{q}, J=3.9 \mathrm{~Hz}), 44.83 ;{ }^{19} \mathrm{~F}$ NMR (376 MHz, $\left.\mathrm{CDCl}_{3}\right) \delta$ : -62.44 ; HRMS calcd for $\mathrm{C}_{12} \mathrm{H}_{11} \mathrm{~N}_{3} \mathrm{~F}_{3}[\mathrm{M}+\mathrm{H}]^{+}$254.0905, found 254.0906.

$\mathrm{N}$-(4-(三氟甲氧基)芐基吡嗪)-2-胺(3s)：黄色固体, $215 \mathrm{mg}$, 产率 80\%. m.p. $75 \sim 76{ }^{\circ} \mathrm{C} ;{ }^{1} \mathrm{H}$ NMR $(400 \mathrm{MHz}$, $\left.\mathrm{CDCl}_{3}\right) \delta: 7.86(\mathrm{dd}, J=2.8,1.5 \mathrm{~Hz}, 1 \mathrm{H}), 7.78(\mathrm{~d}, J=1.6$ $\mathrm{Hz}, 1 \mathrm{H}), 7.68(\mathrm{~d}, J=2.8 \mathrm{~Hz}, 1 \mathrm{H}), 7.32 \sim 7.20(\mathrm{~m}, 2 \mathrm{H})$, 7.08 (d, $J=7.9 \mathrm{~Hz}, 2 \mathrm{H}), 5.37$ (s, 1H), 4.46 (d, $J=5.5 \mathrm{~Hz}$, $2 \mathrm{H}) ;{ }^{13} \mathrm{C}$ NMR (101 MHz, $\left.\mathrm{CDCl}_{3}\right) \delta: 154.40,148.51$ (q, $J=2.1 \mathrm{~Hz}), 141.97,137.45,133.10,132.20,128.87$, 124.29 (q, $J=255.0 \mathrm{~Hz}), 121.22,44.60 ;{ }^{19} \mathrm{~F}$ NMR $(376$ $\left.\mathrm{MHz}, \mathrm{CDCl}_{3}\right) \delta$ : -57.93 ; HRMS calcd for $\mathrm{C}_{12} \mathrm{H}_{11} \mathrm{~N}_{3} \mathrm{~F}_{3} \mathrm{O}$ $[\mathrm{M}+\mathrm{H}]^{+}$270.0854, found 270.0856 .

$N-$-(3,5-双 (三氟甲基)茮基吡嗪)-2-胺(3t): 白色固体, $250 \mathrm{mg}$, 产率 78\%. m.p. $76 \sim 77{ }^{\circ} \mathrm{C} ;{ }^{1} \mathrm{H}$ NMR $(400 \mathrm{MHz}$, $\left.\mathrm{CDCl}_{3}\right) \delta: 7.95 \sim 7.80(\mathrm{~m}, 2 \mathrm{H}), 7.78 \sim 7.61(\mathrm{~m}, 4 \mathrm{H}), 5.54$ (s, 1H), $4.63(\mathrm{~d}, J=5.8 \mathrm{~Hz}, 2 \mathrm{H}) ;{ }^{13} \mathrm{C}$ NMR $(101 \mathrm{MHz}$, 
$\left.\mathrm{CDCl}_{3}\right) \delta: 154.12,141.99,141.81,133.49,132.58,131.83$ (q, $J=33.3 \mathrm{~Hz}), 127.56(\mathrm{q}, J=3.8 \mathrm{~Hz}), 127.32(\mathrm{q}, J=$ $271.1 \mathrm{~Hz}), 121.36$ (q, $J=4.0 \mathrm{~Hz}), 44.36 ;{ }^{19} \mathrm{~F}$ NMR $(376$ $\left.\mathrm{MHz}, \mathrm{CDCl}_{3}\right) \delta$ : -62.96 ; HRMS calcd for $\mathrm{C}_{13} \mathrm{H}_{10} \mathrm{~N}_{3} \mathrm{~F}_{6}$ $[\mathrm{M}+\mathrm{H}]^{+} 322.0779$, found 322.0780 .

$N$-(3,5-二甲氧基苄基吡嗪)-2-胺(3u): 黄色固体, $213 \mathrm{mg}$, 产率 87\%. m.p. 103 104 ${ }^{\circ} \mathrm{C} ;{ }^{1} \mathrm{H}$ NMR $(400$ $\left.\mathrm{MHz}, \mathrm{CDCl}_{3}\right) \delta: 8.02 \sim 7.91(\mathrm{~m}, 1 \mathrm{H}), 7.86(\mathrm{~s}, 1 \mathrm{H}), 7.77(\mathrm{~d}$, $J=2.8 \mathrm{~Hz}, 1 \mathrm{H}), 6.48(\mathrm{~d}, J=2.4 \mathrm{~Hz}, 2 \mathrm{H}), 6.36$ (t, $J=2.3$ $\mathrm{Hz}, 1 \mathrm{H}), 5.46$ (s, 1H), 4.46 (d, $J=5.3 \mathrm{~Hz}, 2 \mathrm{H}), 3.73$ (s, $6 \mathrm{H}) ;{ }^{13} \mathrm{C}$ NMR (101 MHz, $\left.\mathrm{CDCl}_{3}\right) \delta: 161.09,154.56$, $141.95,141.05,132.90,132.16,105.42,99.20,55.29$, 45.52; HRMS calcd for $\mathrm{C}_{13} \mathrm{H}_{16} \mathrm{~N}_{3} \mathrm{O}_{2}[\mathrm{M}+\mathrm{H}]^{+}$246.1243, found 246.1245 .

$N$-(苯并 $[d][1,3]$ 二氧杂环-5-基甲基)-2-胺(3v): 黄色 固体, $183 \mathrm{mg}$, 产率 $80 \%$. m.p. $106 \sim 107{ }^{\circ} \mathrm{C} ;{ }^{1} \mathrm{H}$ NMR $\left(400 \mathrm{MHz}, \mathrm{CDCl}_{3}\right) \delta: 7.89 \sim 7.81(\mathrm{~m}, 1 \mathrm{H}), 7.75(\mathrm{~s}, 1 \mathrm{H})$, $7.67(\mathrm{~d}, J=2.9 \mathrm{~Hz}, 1 \mathrm{H}), 6.81 \sim 6.58(\mathrm{~m}, 3 \mathrm{H}), 5.81(\mathrm{~s}, 2 \mathrm{H})$, $5.32(\mathrm{~s}, 1 \mathrm{H}), 4.33(\mathrm{~d}, J=5.2 \mathrm{~Hz}, 2 \mathrm{H}) ;{ }^{13} \mathrm{C}$ NMR $(101 \mathrm{MHz}$, $\left.\mathrm{CDCl}_{3}\right) \delta: 154.49,147.92,146.92,141.96,132.86,132.41$, $132.15,120.79,108.34,108.14,101.07,45.26$; HRMS calcd for $\mathrm{C}_{12} \mathrm{H}_{12} \mathrm{~N}_{3} \mathrm{O}_{2}[\mathrm{M}+\mathrm{H}]^{+}$230.0930, found 230.0928 .

$N$-(噻吩-2-基甲基吡嗪)-2-胺(3w): 黄色固体, 162 $\mathrm{mg}$, 产率 85\%. m.p. $69 \sim 70{ }^{\circ} \mathrm{C}$; ${ }^{1} \mathrm{H}$ NMR $(400 \mathrm{MHz}$, $\left.\mathrm{CDCl}_{3}\right) \delta: 7.92(\mathrm{dd}, J=2.8,1.5 \mathrm{~Hz}, 1 \mathrm{H}), 7.80(\mathrm{~d}, J=1.6$ $\mathrm{Hz}, 1 \mathrm{H}), 7.73(\mathrm{~d}, J=2.9 \mathrm{~Hz}, 1 \mathrm{H}), 7.12(\mathrm{dd}, J=5.1,1.3 \mathrm{~Hz}$, $1 \mathrm{H}), 6.93$ (dq, $J=2.2,1.0 \mathrm{~Hz}, 1 \mathrm{H}), 6.87$ (dd, $J=5.1,3.5$ $\mathrm{Hz}, 1 \mathrm{H}), 5.20$ (s, 1H), 4.65 (d, $J=4.7 \mathrm{~Hz}, 2 \mathrm{H}) ;{ }^{13} \mathrm{C} \mathrm{NMR}$ $\left(101 \mathrm{MHz}, \mathrm{CDCl}_{3}\right) \delta$ : 154.07, 141.91, 141.62, 133.25, 132.49, 126.93, 125.77, 125.03, 40.32; HRMS calcd for $\mathrm{C}_{9} \mathrm{H}_{10} \mathrm{~N}_{3} \mathrm{~S}[\mathrm{M}+\mathrm{H}]^{+}$192.0595, found 192.0597.

$N$-(萗-1-基甲基吡嗪)-2-胺 $(\mathbf{3 x}$ ): 淡黄色固体, 228 $\mathrm{mg}$, 产率 90\%. m.p. $112 \sim 113{ }^{\circ} \mathrm{C} ;{ }^{1} \mathrm{H}$ NMR $(400 \mathrm{MHz}$, $\left.\mathrm{CDCl}_{3}\right) \delta: 7.86 \sim 7.80(\mathrm{~m}, 1 \mathrm{H}), 7.80 \sim 7.73(\mathrm{~m}, 1 \mathrm{H})$, $7.71 \sim 7.65(\mathrm{~m}, 1 \mathrm{H}), 7.61(\mathrm{~d}, J=8.1 \mathrm{~Hz}, 1 \mathrm{H}), 7.60 \sim 7.53$ $(\mathrm{m}, 2 \mathrm{H}), 7.37 \sim 7.29(\mathrm{~m}, 2 \mathrm{H}), 7.28(\mathrm{~d}, J=7.6 \mathrm{~Hz}, 1 \mathrm{H})$, 7.23 (t, $J=7.5 \mathrm{~Hz}, 1 \mathrm{H}), 5.21(\mathrm{t}, J=5.3 \mathrm{~Hz}, 1 \mathrm{H}), 4.74$ (d, $J=5.0 \mathrm{~Hz}, 2 \mathrm{H}) ;{ }^{13} \mathrm{C}$ NMR $\left(101 \mathrm{MHz}, \mathrm{CDCl}_{3}\right) \delta: 154.49$, $141.94,133.91,133.74,132.65,132.42,131.52,128.85$, $128.49,126.52,126.23,126.02,125.50,123.52,43.53$; HRMS calcd for $\mathrm{C}_{15} \mathrm{H}_{14} \mathrm{~N}_{3}[\mathrm{M}+\mathrm{H}]^{+}$236.1188, found 236.1186 .
3.2.2 TNP-Cu@ $\mathrm{rGO}$ 催化的醇与酮的借氢反应实验 方法

在 $25 \mathrm{~mL}$ 干燥反应管中, 依次加入苯甲醇(2a) (119 $\mathrm{mg}, 1.1 \mathrm{mmol}) 、$ 苯乙酮(5a)(120 mg, $1.0 \mathrm{mmol}) 、$ 催化剂 TNP-Cu@rGO (15 mg, 3 wt \%)、KOH (42 mg, 0.75 $\mathrm{mmol})$ 、甲苯 $(2.0 \mathrm{~mL})$ 于 $110{ }^{\circ} \mathrm{C}$ 油浴锅中搅拌反应 $12 \mathrm{~h}$, 反应过程中用 TLC 板追踪反应进度, 反应结束后, 待其 冷却至室温, 将反应物中加入水淬灭并加入乙酸乙酯 (5 $\mathrm{mL} \times 3$ )萃取, 合并有机相并用无水 $\mathrm{MgSO}_{4}$ 干燥, 减压 浓缩得到粗产品并采用柱层析纯化 $[V$ (石油醚) : $V$ (二氯 甲烷 $=20: 1$ ], 得到 3-苯基苯丙酮 $(\mathbf{6 a})$ : 白色固体, 180 mg, 产率 86\%. m.p. 69 70 ${ }^{\circ} \mathrm{C}$ (Ref. ${ }^{[14 a]} 69 \sim 70{ }^{\circ} \mathrm{C}$ ); ${ }^{1} \mathrm{H}$ NMR (400 MHz, $\left.\mathrm{CDCl}_{3}\right) \delta: 8.07 \sim 7.98(\mathrm{~m}, 2 \mathrm{H}), 7.61(\mathrm{~s}$, $1 \mathrm{H}), 7.54 \sim 7.48(\mathrm{~m}, 2 \mathrm{H}), 7.42 \sim 7.32(\mathrm{~m}, 4 \mathrm{H}), 7.28(\mathrm{~s}$, $1 \mathrm{H}), 3.36(\mathrm{dd}, \quad J=8.7,7.0 \mathrm{~Hz}, 2 \mathrm{H}), 3.15(\mathrm{dd}, J=8.5,6.9$ $\mathrm{Hz}, 2 \mathrm{H}) ;{ }^{13} \mathrm{C}$ NMR $\left(101 \mathrm{MHz}, \mathrm{CDCl}_{3}\right) \delta: 199.20,141.39$, $136.98,133.11,128.68,128.61,128.52,128.12,126.22$, 40.47, 30.22; HRMS calcd for $\mathrm{C}_{15} \mathrm{H}_{15} \mathrm{O} \quad[\mathrm{M}+\mathrm{H}]^{+}$ 211.1123, found 211.1124.

3-(4-甲基苯基)-1-苯丙烷-1-酮(6b): 黄色固体，186 mg, 产率 83\%. m.p. 33 34 ${ }^{\circ} \mathrm{C}$ (Ref. ${ }^{[14 a]} 34 \sim 35{ }^{\circ} \mathrm{C}$ ); ${ }^{1} \mathrm{H}$ NMR $\left(400 \mathrm{MHz}, \mathrm{CDCl}_{3}\right) \delta: 7.98(\mathrm{~d}, J=7.0 \mathrm{~Hz}, 2 \mathrm{H})$, $7.60 \sim 7.55(\mathrm{~m}, 1 \mathrm{H}), 7.48(\mathrm{dd}, J=8.3,7.0 \mathrm{~Hz}, 2 \mathrm{H}), 7.16(\mathrm{q}$, $J=8.1 \mathrm{~Hz}, 4 \mathrm{H}), 3.34 \sim 3.28(\mathrm{~m}, 2 \mathrm{H}), 3.08 \sim 3.03(\mathrm{~m}, 2 \mathrm{H})$, 2.35 (s, 3H); ${ }^{13} \mathrm{C}$ NMR $\left(101 \mathrm{MHz}, \mathrm{CDCl}_{3}\right) \delta: 199.37$, 138.20, 136.91, 135.64, 133.04, 129.22, 128.61, 128.30, 128.06, 40.63, 29.74, 21.01; HRMS calcd for $\mathrm{C}_{16} \mathrm{H}_{17} \mathrm{O}$ $[\mathrm{M}+\mathrm{H}]^{+}$225.1279, found 225.1279.

3-(4-甲氧基苯基)-1-苯丙烷-1-酮(6c)：白色固体， $192 \mathrm{mg}$, 产率 80\%. m.p. 59 60 ${ }^{\circ} \mathrm{C}$ (Ref. ${ }^{[14 a]}$ 59 $\left.60{ }^{\circ} \mathrm{C}\right) ;{ }^{1} \mathrm{H}$ NMR $\left(400 \mathrm{MHz}, \mathrm{CDCl}_{3}\right) \delta: 8.02 \sim 7.95(\mathrm{~m}$, $2 \mathrm{H}), 7.57(\mathrm{~d}, J=7.4 \mathrm{~Hz}, 1 \mathrm{H}), 7.48(\mathrm{t}, J=7.7 \mathrm{~Hz}, 2 \mathrm{H})$, $7.23 \sim 7.17(\mathrm{~m}, 2 \mathrm{H}), 6.91 \sim 6.85(\mathrm{~m}, 2 \mathrm{H}), 3.82(\mathrm{~s}, 3 \mathrm{H})$, $3.30(\mathrm{dd}, J=8.4,6.9 \mathrm{~Hz}, 2 \mathrm{H}), 3.05(\mathrm{t}, J=7.7 \mathrm{~Hz}, 2 \mathrm{H}) ;{ }^{13} \mathrm{C}$ NMR $\left(101 \mathrm{MHz}, \mathrm{CDCl}_{3}\right) \delta: 197.81,163.48,141.50$, $130.32,130.04,128.52,128.44,126.09,113.76,55.46$, 40.11, 30.37; HRMS calcd for $\mathrm{C}_{16} \mathrm{H}_{17} \mathrm{O}_{2}[\mathrm{M}+\mathrm{H}]^{+}$ 241.1229, found 241.1230.

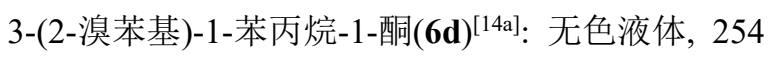
$\mathrm{mg}$, 产率 $88 \% .{ }^{1} \mathrm{H}$ NMR $\left(400 \mathrm{MHz}, \mathrm{CDCl}_{3}\right) \delta: 8.01(\mathrm{~d}$, $J=7.0 \mathrm{~Hz}, 2 \mathrm{H}), 7.58(\mathrm{dd}, J=7.9,1.5 \mathrm{~Hz}, 2 \mathrm{H}), 7.48(\mathrm{t}, J=$ $7.6 \mathrm{~Hz}, 2 \mathrm{H}), 7.35$ (dd, $J=7.7,1.8 \mathrm{~Hz}, 1 \mathrm{H}), 7.27$ (td, $J=$ 7.4, $1.3 \mathrm{~Hz}, 1 \mathrm{H}), 7.11(\mathrm{td}, J=7.6,1.8 \mathrm{~Hz}, 1 \mathrm{H}), 3.39 \sim 3.31$ (m, 2H), $3.22(\mathrm{dd}, J=8.9,6.9 \mathrm{~Hz}, 2 \mathrm{H}) ;{ }^{13} \mathrm{C}$ NMR $(101$ $\left.\mathrm{MHz}, \mathrm{CDCl}_{3}\right) \delta: 198.91,140.60,136.81,133.14,132.92$, 
130.83, 128.64, 128.10, 128.01, 127.67, 124.40, 38.63, 30.83; HRMS calcd for $\mathrm{C}_{15} \mathrm{H}_{14} \mathrm{BrO}[\mathrm{M}+\mathrm{H}]^{+}$289.0228, found 289.0227 .

1-苯基-3-(噻吩-2-基)丙烷-1-酮(6e $)^{[14 a]}$ ：黄色液体, $175 \mathrm{mg}$, 产率 $81 \% .{ }^{1} \mathrm{H} \mathrm{NMR}\left(400 \mathrm{MHz}, \mathrm{CDCl}_{3}\right) \delta: 8.04 \sim$ $7.97(\mathrm{~m}, 2 \mathrm{H}), 7.63 \sim 7.56(\mathrm{~m}, 1 \mathrm{H}), 7.53 \sim 7.46(\mathrm{~m}, 2 \mathrm{H})$, $7.16(\mathrm{dd}, J=5.1,1.2 \mathrm{~Hz}, 1 \mathrm{H}), 6.96(\mathrm{dd}, J=5.1,3.4 \mathrm{~Hz}$, $1 \mathrm{H}), 6.92 \sim 6.87(\mathrm{~m}, 1 \mathrm{H}), 3.43 \sim 3.37(\mathrm{~m}, 2 \mathrm{H}), 3.36 \sim 3.30$ $(\mathrm{m}, 2 \mathrm{H}) ;{ }^{13} \mathrm{C} \mathrm{NMR}\left(101 \mathrm{MHz}, \mathrm{CDCl}_{3}\right) \delta: 198.59,143.91$, $136.79,133.18,128.66,128.06,126.88,124.70,123.40$, 40.46, 24.17; HRMS calcd for $\mathrm{C}_{13} \mathrm{H}_{13} \mathrm{SO}[\mathrm{M}+\mathrm{H}]^{+}$ 217.0687 , found 217.0686 .

1-(4-氯苯基)-3-苯丙烷-1-酮(6f): 黄色固体, $172 \mathrm{mg}$, 产率 77\%. m.p. $76 \sim 78{ }^{\circ} \mathrm{C}$ (Ref. ${ }^{[14 a]} 76 \sim 78{ }^{\circ} \mathrm{C}$ ); ${ }^{1} \mathrm{H}$ NMR (400 MHz, $\left.\mathrm{CDCl}_{3}\right) \delta: 7.92(\mathrm{~d}, J=8.6 \mathrm{~Hz}, 2 \mathrm{H}), 7.45$ $(\mathrm{d}, J=8.6 \mathrm{~Hz}, 2 \mathrm{H}), 7.35 \sim 7.31(\mathrm{~m}, 2 \mathrm{H}), 7.27$ (dd, $J=8.2$, $1.5 \mathrm{~Hz}, 3 \mathrm{H}), 3.33 \sim 3.27(\mathrm{~m}, 2 \mathrm{H}), 3.09(\mathrm{dd}, J=8.5,6.8 \mathrm{~Hz}$, $2 \mathrm{H}) ;{ }^{13} \mathrm{C} \mathrm{NMR}\left(101 \mathrm{MHz}, \mathrm{CDCl}_{3}\right) \delta: 198.00,141.07$, $139.52,135.20,129.47,128.94,128.58,128.42,126.24$, 40.43, 30.07; HRMS calcd for $\mathrm{C}_{15} \mathrm{H}_{14} \mathrm{ClO}[\mathrm{M}+\mathrm{H}]^{+}$ 245.0733, found 245.0734.

1-(3-苯基-1-(间甲苯基)丙烷-1-酮 $(6 \mathbf{g})^{[14 b]}$ : 黄色油 状液体, $177 \mathrm{mg}$, 产率 79\%. ${ }^{1} \mathrm{H}$ NMR (400 $\left.\mathrm{MHz}, \mathrm{CDCl}_{3}\right)$ $\delta: 7.79(\mathrm{~d}, J=7.2 \mathrm{~Hz}, 2 \mathrm{H}), 7.42 \sim 7.26(\mathrm{~m}, 7 \mathrm{H}), 3.32(\mathrm{dd}$, $J=8.6,6.9 \mathrm{~Hz}, 2 \mathrm{H}), 3.10(\mathrm{dd}, J=8.5,6.9 \mathrm{~Hz}, 2 \mathrm{H}), 2.43$ (s, $3 \mathrm{H}) ;{ }^{13} \mathrm{C} \mathrm{NMR}\left(101 \mathrm{MHz}, \mathrm{CDCl}_{3}\right) \delta: 199.47,141.39$, $138.41,136.94,133.83,129.06,128.61,128.54,128.45$, 126.13, 125.27, 40.54, 30.20, 21.37; HRMS calcd for $\mathrm{C}_{16} \mathrm{H}_{17} \mathrm{O}[\mathrm{M}+\mathrm{H}]^{+}$225.1279, found 225.1280.

1-(3-甲氧基苯基)-3-苯丙烷-1-酮(6h) ${ }^{[14 a]}$ ：黄色油状 液体, $197 \mathrm{mg}$, 产率 $82 \% .{ }^{1} \mathrm{H}$ NMR $\left(400 \mathrm{MHz}, \mathrm{CDCl}_{3}\right) \delta$ : $7.61 \sim 7.51(\mathrm{~m}, 2 \mathrm{H}), 7.42 \sim 7.23(\mathrm{~m}, 6 \mathrm{H}), 7.14(\mathrm{ddd}, J=$ 8.2, 2.7, $1.0 \mathrm{~Hz}, 1 \mathrm{H}), 3.88$ (s, 3H), 3.33 (dd, $J=8.5,6.9$ $\mathrm{Hz}, 2 \mathrm{H}), 3.11(\mathrm{dd}, J=8.4,6.9 \mathrm{~Hz}, 2 \mathrm{H}) ;{ }^{13} \mathrm{C} \mathrm{NMR}(101$ $\left.\mathrm{MHz}, \mathrm{CDCl}_{3}\right) \delta: 199.04,159.91,141.32,138.31,129.61$, $128.56,128.46,126.17,120.70,119.57,112.36,55.45$, 40.56, 30.23; HRMS calcd for $\mathrm{C}_{16} \mathrm{H}_{17} \mathrm{O}_{2}[\mathrm{M}+\mathrm{H}]^{+}$ 241.1229 , found 241.1228 .

\section{辅助材料(Supporting Information) 产物 $\mathbf{3 a} \sim \mathbf{3 x}, \mathbf{6 a} \sim$} 6h 的核磁共振氢谱、碳谱和氟谱. 这些材料可以免费从 本刊网站(http://sioc-journal.cn/)上下载.

\section{References}

[1] (a) Cai, L.; Zhang, K.; Chen, S.; Lepage, R. J.; Houk, K. N.; Krenske, E. H.; Kwon, O. J. Am. Chem. Soc. 2019, 141, 9537. (b) Ding, B.; Jiang, Y.; Zhang, Y.; Ye, R.; Sun, J.; Yan, C. Chin. J. Org. Chem. 2020, 40, 1003 (in Chinese).

(丁邦东，姜业朝，张瑜，叶蓉，孙晶，颜朝国，有机化学，2020 40, 1003.)

(c) Wen, D.; Zheng, Q.; Wang, C.; Tu, T. Org. Lett. 2021, 23, 3718.

(d) Zhou, Y.; Zhao, Z.; Zeng, L.; Li, M.; He, Y.; Gu, L. Chin. J. Org. Chem. 2021, 41, 1072 (in Chinese)

(周娅琴, 赵志恒, 曾亮, 李鸣, 何永辉, 谷利军, 有机化学, 2021, 41, 1072.)

(e) Xie, R.; Lu, G.-P.; Jiang, H.-F.; Zhang, M. J. Catal. 2020, 383, 239.

(d) Liu, Y.; Meng, J.; Li, C.; Lin, L.; Xu, Y. Chin. J. Org. Chem. 2020, 40, 2742 (in Chinese).

(刘颖杰，孟建萍，李晨，林立青，许颖，有机化学，2021，40, 2742.)

[2] (a) Yu, H.; Zhang, R.; Yang, F.; Xie, Y.; Guo, Y.; Yao, W.; Zhou, W. Trends Food Sci. Technol. 2021, 112, 795.

(b) Chen, J.; Wang, Y.; Yu, J.; Cheng, J.; Zheng, H. Chin. J. Org. Chem. 2020, 40, 78 (in Chinese).

(陈晶晶，王莹淑，余珺，成佳佳，郑辉东，有机化学，2020，40, 78.

(c) Wang, F.; Wei, M.; Duan, X.; Liu, X.; Yao, S.; Wang, J.; Zhu, H.; Chen, C.; Gu, L.; Zhang, Y. Org. Chem. Front. 2020, 7, 3616.

(d) Zhang, H. J.; Yang, Z. P.; Gu, Q.; You, S. L. Org. Lett. 2019, 21, 3314.

[3] (a) Hassan, N. W.; Saudi, M. N.; Abdel-Ghany, Y. S.; Ismail, A.; Elzahhar, P. A.; Sriram, D.; Nassra, R.; Abdel-Aziz, M. M. Bioorg. Chem. 2020, 96, 103610 .

(b) Kučerová-Chlupáčová, M.; Opletalová, V.; Jampílek, J.; Doležel, J.; Dohnal, J.; Pour, M.; Kuneš, J.; Voříšek, V. Collect. Czech. Chem. C 2008, 73, 1 .

(c) Wang, N.; Mani, A.; Chen, X.; Wang, B.; Chen, S.; Yao, C.; Wang, Z. Chin. J. Org. Chem. 2019, 39, 2771 (in Chinese).

(王能，Arulkumar Mani，陈孝云，王柏文，陈思鸿，姚辰，汪朝 阳, 有机化学, 2019, 39, 2771.)

(d) Liu, J.; Xie, Y.; Yang, Q.; Huang, N.; Wang, L. Chin. J. Org. Chem. 2021, 41, 2374 (in Chinese).

(刘金妮，谢益碧，阳青青，黄年玉，王龙，有机化学，2021，41, 2374.)

(e) Tian, A.-Q.; Luo, X.-H.; Ren, Z.-L.; Zhao, J.; Wang, L. New J. Chem. 2021, 45, 9614.

(f) Yu, S.-Q.; Liu, N.; Liu, M.-G.; Wang, L. J. Chem. Res. 2021, 45, 237.

[4] (a) Wei, R.; Bao, H. Chin. J. Org. Chem. 2020, 40, 1797 (in Chinese).

(韦榕标, 鲍红丽, 有机化学, 2020, 40, 1797.)

(b) Navarro, O.; Marion, N.; Mei, J.; Nolan, S. P. Chem. Eur. J. 2006, 12,5142 .

(c) Wolfe, J. P.; Buchwald, S. L. J. Am. Chem. Soc. 1997, 119, 6054.

(d) Mizuta, T.; Sakaguchi, S.; Ishii, Y. J. Org. Chem. 2005, 70, 2195.

(4e) Vernin, G.; Hornwood, M. P.-E. Chichester 1982, 305, 70

[5] (a) Hao, S.; Yang, J.; Liu, P.; Xu, J.; Yang, C.; Li, F. Org. Lett. 2021, 23. 2553.

(b) Liu, P.; Yang, J.; Ai, Y.; Hao, S.; Chen, X.; Li, F. J. Catal. 2021, 396, 281.

(c) Wei, D.; Yang, P.; Yu, C.; Zhao, F.; Wang, Y.; Peng, Z. J. Org Chem. 2021, 86, 2254.

(d) Meng, C.; Liu, P.; Tung, N. T.; Han, X.; Li, F. J. Org. Chem 2020, $85,5815$.

(e) Zhu, Z.-H.; Li, Y.; Wang, Y.-B.; Lan, Z.-G.; Zhu, X.; Hao, X.-Q. Organometallics 2019, 38, 2156.

[6] (a) Li, F.; Xie, J.; Shan, H.; Sun, C.; Chen, L. RSC Adv. 2012, 2 , 8645.

(b) Liang, R.; Li, S.; Wang, R.; Lu, L.; Li, F. Org. Lett. 2017, 19, 5790

(c) Fujita, K.-I.; Toyooka, G.; Tuji, A. Synthesis 2018, 50, 4617. 
(d) Nallagangula, M.; Sujatha, C.; Bhat, V. T.; Namitharan, K. Chem. Commun. 2019, 55, 8490.

(e) Sankar, V.; Kathiresan, M.; Sivakumar, B.; Mannathan, S. Adv. Synth. Catal. 2020, 362, 4409.

[7] (a) Cao, L.; Zhao, H.; Tan, Z.; Guan, R.; Jiang, H.; Zhang, M. Org Lett. 2020, 22, 4781 .

(b) Tan, Z.; Ci, C.; Yang, J.; Wu, Y.; Cao, L.; Jiang, H.; Zhang, M. ACS Catal. 2020, 10, 5243.

(c) Xie, F.; Li, Y.; Chen, X.; Chen, L.; Zhu, Z.; Li, B.; Huang, Y.; Zhang, K.; Zhang, M. Chem. Commun. 2020, 56, 5997.

(d) Wu, D.; Cheng, X.; Liu, Y.; Cheng, G.; Guan, X.; Deng, Q. Chin. J. Org. Chem. 2020, 40, 3362 (in Chinese).

(吴敦奇, 成轩, 刘炎开, 成果, 管笑宇, 邓清海, 有机化学, 2020, 40, 3362.)

(e) Zhang, G.; Jiang, Y.; Ding, C.; Hou, X. Chin. J. Org. Chem. 2020, 40, 3399 (in Chinese).

(张高鹏, 江阳杰, 丁昌华, 侯雪龙, 有机化学, 2020, 40, 3399.)

(f) Cao, Z.; Qiao, H.; Zeng, F. Organometallics 2019, 38, 797.

(g) Li, S.; Li, X.; Li, Q.; Yuan, Q.; Shi, X.; Xu, Q. Green Chem. 2015, 17, 3260 .

[8] (a) Balamurugan, G.; Ramesh, R.; Malecki, J. G. J. Org. Chem. 2020, 85,7125 .

(b) Liu, Z.; Zhang, X.; Zhang, H.; Jiang, H.; Zhao, X.; Shi, L.; Zhu, X.; Hao, X.; Song, M. Chin. J. Org. Chem. 2020, 40, 2755 (in Chinese).

(刘子琳, 张小洁, 张恒, 姜辉, 赵雪梅, 石林林, 朱新举, 郝新 奇, 宋毛平, 有机化学, 2020, 40, 2755.)

(c) Xie, F.; Li, Y.; Chen, X.; Chen, L.; Zhu, Z.; Li, B.; Huang, Y.; Zhang, K.; Zhang, M. Chem. Commun. 2020, 56, 5997.

[9] (a) Yao, W.; Zhang, Y.; Zhu, H.; Ge, C.; Wang, D. Chin. Chem. Lett. 2020, 31, 701 .

(b) Cao, F.; Duan, Z.-C.; Zhu, H.; Wang, D. Mol. Catal. 2021, 503, 111391. (c) Xu, Z.; Yu, X.; Sang, X.; Wang, D. Green Chem. 2018, 20, 2571.

(d) Yang, Q.; Zhang, Y.; Zeng, W.; Duan, Z.-C.; Sang X.; Wang, D. Green Chem. 2019, 21, 5683.

[10] (a) Tao, R.; Yang, Y.; Zhu, H.; Hu, X.; Wang, D. Green Chem. 2020, 22,8452 .

(b) Zhu, G.; Duan, Z.-C.; Zhu, H.; Qi, M.; Wang, D. Mol. Catal. 2021, 505, 111516

(c) Zhu, G.; Duan, Z.-C.; Zhu, H.; Ye, D.; Wang, D. Chin. Chem. Lett. 2021, 32, 10.1016/j.cclet.2021.06.060.

(d) Hu, X.; Yang, B.; Yao, W.; Wang, D. Chin. J. Org. Chem. 2018, 38, 3296 (in Chinese).

(胡昕宇, 杨伯斌, 姚玮, 王大伟, 有机化学, 2018, 38, 3296.)

[11] Hu, W.; Zhang, Y.; Zhu, H.; Ye, D.; Wang, D. Green. Chem. 2019, $21,5345$.

[12] Yang, F.-L.; Wang, Y.-H.; Ni, Y.-F.; Gao, X.; Song, B.; Zhu, X.; Hao, X.-Q. Eur. J. Org. Chem. 2017, 2017, 3481.

[13] (a) Donthiri, R. R.; Pappula, V.; Mohan, D. C.; Gaywala, H. H.; Adimurthy, S. J. Org. Chem. 2013, 78, 6775.

(b) Li, S.; Li, X.; Li, Q.; Yuan, Q.; Shi, X.; Xu, Q. Green Chem. 2015, 17, 3260 .

(c) Walsh, K.; Sneddon, H. F.; Moody, C. J. ChemSusChem 2013, 6, 1455 .

(e) Duron, S. G.; Lindstrom, A.; Bonnefous, C.; Zhang, H.; Chen, X.; Symons, K. T.; Sablad, M.; Rozenkrants, N.; Zhang, Y.; Wang, L.; Yazdani, N.; Shiau, A. K.; Noble, S. A.; Rix, P.; Rao, T. S.; Hassig, C. A.; Smith, N. D. Bioorg. Med. Chem. Lett. 2012, 22, 1237.

[14] (a) Cao, X. N.; Wan, X. M.; Yang, F. L.; Li, K.; Hao, X. Q.; Shao, T.; Zhu, X.; Song, M. P. J. Org. Chem. 2018, 83, 3657.

(b) Liu, P.; Liang, R.; Lu, L.; Yu, Z.; Li, F. J. Org. Chem. 2017, 82, 1943.

(Cheng, F.) 